[Article]

\title{
八硝基立方烷高温热分解分子动力学模拟
}

\author{
杨 镇 何远航 ${ }^{*}$ \\ (北京理工大学, 爆炸与科学国家重点实验室, 北京 100081)
}

\begin{abstract}
摘要: 随着对高能量密度材料的性能要求不断提高, 新型高能量密度材料成为近期研究热点, 其中八硝基立 方烷(ONC)由于其优越的性能成为其中典型的代表, 然而关于八硝基立方烷热分解的动力学机理研究比较 少。本文采用ReaxFF 反应力场模拟高温条件下凝聚相八硝基立方烷初始热分解过程。研究发现热分解过程 中八硝基立方烷笼状骨架结构中 $\mathrm{C}-\mathrm{C}$ 键最先发生断裂, 并逐步破坏形成八硝基环辛烯等, 随后出现 $\mathrm{NO}_{2}$ 和 $\mathrm{O}$ 等, 计算结果表明笼状骨架结构的破坏存在三种不同路径。八硝基立方烷在高温条件下热分解的主要产物 有 $\mathrm{NO}_{2} 、 \mathrm{O}_{2} 、 \mathrm{CO}_{2} 、 \mathrm{~N}_{2} 、 \mathrm{NO}_{3} 、 \mathrm{NO} 、 \mathrm{CNO}$ 以及 $\mathrm{CO}$ 等, 其中 $\mathrm{N}_{2}$ 和 $\mathrm{CO}_{2}$ 是终态产物, 不同温度对产物均产生 不同程度的影响。
\end{abstract}

关键词: ReaxFF; 高温热分解; 分子动力学; 八硝基立方烷; 反应机理

中图分类号: 0643; 0642

\section{Pyrolysis of Octanitrocubane via Molecular Dynamics Simulations}

\author{
YANG Zhen HE Yuan-Hang*
}

(State Key Laboratory of Explosion Science and Technology, Beijing Institute of Technology, Biejing 100081, P. R. China)

\begin{abstract}
As the requirements for the performance of high-energy-density materials increase, research to develop new types of high-energy-density materials has become highly heated recently. Octanitrocubane, by virtue of its superior performance, is one of the typical representatives of recently developed high-energy-density materials. However, there have been few studies on the thermal decomposition mechanism of octanitrocubane, even though they are essential to analyze the thermostability and sensitivity of octanitrocubane, as well as to achieve its efficient application. In this study, the initial pyrolysis process of condensed-phase octanitrocubane at high temperature was investigated using ReaxFF reactive molecular dynamics simulation. The results showed that it is the $\mathrm{C}-\mathrm{C}$ bond of the octanitrocubane cage skeleton structure that breaks first, and then octanitrocubane cage skeleton structure is gradually destroyed, and the small molecules such as $\mathrm{NO}_{2}$ and $\mathrm{O}$ occur afterwards. The simulation identified three different damage pathways of the cage skeleton. The main products of octanitrocubane thermal decomposition at high temperature are $\mathrm{NO}_{2}, \mathrm{O}_{2}, \mathrm{CO}_{2}, \mathrm{~N}_{2}, \mathrm{NO}_{3}, \mathrm{NO}, \mathrm{CNO}$, and $\mathrm{CO}$, of which $\mathrm{N}_{2}$ and $\mathrm{CO}_{2}$ are the final products. The products that form depend on temperature.
\end{abstract}

Key Words: ReaxFF; Pyrolysis; Molecular dynamics; Octanitrocubane; Reaction mechanism

\section{1 引言}

随着武器系统的不断进步对含能材料的性能 提出了更高的要求, 高能量密度材料成为近期研 究热点。国内外学者对新型高能量密度材料进行 设计、合成以及理论研究, 90 年代合成的六硝基
六氮杂异伍兹烷(CL-20)为其中的典型代表。多硝 基立方烷不仅具有高密度、高焓值、高爆速、高 爆压而且具有较低的感度, 而八硝基立方烷(ONC) 在多硝基立方烷中爆轰性能最好, 明显优于三硝 基甲苯(TNT)、黑索金(RDX)和奥克托今(HMX)等

Received: October 14, 2015; Revised: December 24, 2015; Published on Web: December 25, 2015.

${ }^{*}$ Corresponding author. Email: heyuanhang@bit.edu.cn; Tel: +86-10-68918878

(c) Editorial office of Acta Physico-Chimica Sinica 
常规炸药 ${ }^{1-3}$ 。ONC 是继 CL-20 之后又一优良高能 量密度材料, 将来可以应用于炸药和固体火箭推 进剂等方面, 具有很好的发展前景。

1964 年 Eaton 和 $\mathrm{Cole}^{4}$ 首次合成了立方烷 $(\mathrm{CH})_{8}$, 它的骨架是一个立方体结构。同年 Eaton 和 Cole ${ }^{5}$ 首次合成了立方烷的衍生物。在 1984 年, Eaton 以 1,4-二羧酸立方烷为原料, 首次合成了 1,4二硝基立方烷。随后在九十年代分别合成了多硝 基立方烷 ${ }^{6-8}$, 然而, 由于合成 $\mathrm{ONC}$ 的过程极其复 杂, 直到 2000 年Zhang 等 ${ }^{9}$ 才合成 $O N C$ 。近期对多 硝基立方烷研究成为了热点, Richard和 Ball ${ }^{10,11}$ 对 多硝基立方烷的生成焓进行了研究, Peköz 和 Erkoç $^{12}$ 对 ONC 及多硝基立方烷的分解机理及其频 率因子、活化能特征参数 ${ }^{13,14}$ 和爆轰性能 ${ }^{15,16}$ 进行了 研究, 结果表明: 多硝基立方烷最初反应是笼状 结构中的 $\mathrm{C}-\mathrm{C}$ 键发生断裂, $\mathrm{ONC}$ 的冲击稳定性优 于部分硝化立方烷, $\mathrm{ONC}$ 在爆速和爆压方面也优 于多硝基立方烷和传统炸药。然而目前对 ONC 初 始热分解过程及其机理研究比较少。

高能量密度材料爆轰中热分解反应机理的研 究对于高能量密度材料具有特别重要的意义, 尤 其初始反应对于材料的热稳定性和感度具有直接 的作用。由于热分解过程是大量的物理和化学联 同作用的结果, 受多种因素影响。然而初始热分 解过程所涉及微观结构的变化、反应路径、过渡 态、反应机理以及初始产物和中间产物等关键的 化学问题, 在实验中难以实现。ReaxFF反应力场 通过对数百万原子组成的凝聚态含能材料进行模 拟, 能够从微观层面揭示化学反应过程及其机 理, 弥补了实验的不足。

目前, ReaxFF 反应力场已经应用于热分解、 燃烧和催化等 ${ }^{17-22}$ 方面的研究中, Strachan 等 ${ }^{23}$ 、何 远航与其合作者 ${ }^{24,25}$ 、Zhou 和 Huang ${ }^{26}$ 采用 ReaxFF 反应力场对含能材料的初始热分解进行了研究。 本文采用 LAMMPS Molecular Simulator ${ }^{27}$ 中的 ReaxFF 反应力场程序包对 ONC 的初始热分解过程 进行计算, 分析热分解机理以及温度对热分解过 程的影响。从微观层面揭示爆轰中的热分解机理 以及温度对热分解过程的影响, 有助于分析 $\mathrm{ONC}$ 的热稳定性以及感度, 为 $\mathrm{ONC}$ 的应用提供帮助。

\section{2 计算方法及细节}

本文采用 Zhang 等 ${ }^{9}$ 于 2000 年首次合成的 ONC

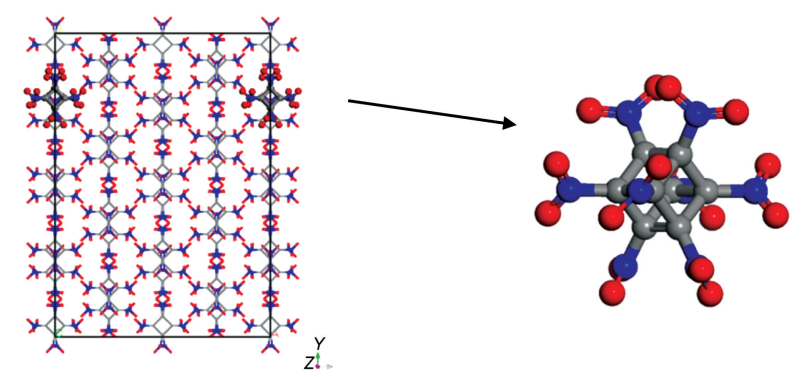

图 1 八硝基立方烷 $(\mathrm{ONC})(2 \times 4 \times 2)$ 晶胞结构 及其分子示意图

Fig.1 Structures of octanitrocubane (ONC) cell $(2 \times 4 \times 2)$ and single molecule color on web version

晶胞参数。为了使模拟工况更接近实际以及准确 的统计产物, 在 $x y z$ 方向建立一个包含 $2 \times 4 \times 2$ 个 晶胞的超胞结构, 该结构包含 64 个 $\mathrm{ONC}$ 分子, 共 计 2048 个原子。图 1 是超胞结构示意图, 其中灰 色原子是碳原子、蓝色原子是氮原子、红色原子 是氧原子, 模拟过程采用周期性边界条件。

在 $300 \mathrm{~K}$ 温度条件下采用 uniform 速度分布器 生成模拟系统中所有原子的初始速度, 然后对系 统进行优化, 获得最小能量结构系统, 初始密度 为 $1.96 \mathrm{~g} \cdot \mathrm{cm}^{-3}$ 。采用等温等压 $(\mathrm{NPT})$ 系综使系统在 $0 \mathrm{~Pa}$ 压力和 $300 \mathrm{~K}$ 温度条件下进行弛豫, 使用 Nose-Hoover 恒压器和 Nose-Hoover 恒温器对压力 和温度进行控制, 弛豫时间为 $5 \mathrm{ps}$ 。经过 $5 \mathrm{ps}$ 弛豫 后, 密度下降到 $1.43 \mathrm{~g} \cdot \mathrm{cm}^{-3}$ 。然后急剧升温, 采 用微正则(NVE)系综, 通过 berenden 恒温器控制系 统温度，分别在 2500、2700、2900、3100、3300 及 $3500 \mathrm{~K}$ 温度条件下进行模拟, 高温持续时间为 $200 \mathrm{ps}$ 。在计算过程中每一步都对原子速度进行调 节, 从而消除迭代计算误差产生的累积效应。通 过耦合参数控制系统温度与热浴温度的耦合程 度, 其中耦合参数和时间步长分别为 10 和 $0.1 \mathrm{fs}$; 键级截断半径为 0.3 , 通过它的大小确定原子间是 否成键, 当任意原子对的键级大于 0.3 时, 则认为 新的化学键形成, 生成新分子, 键级小于 0.3 时, 旧的化学键断裂。

\section{3 结果与分析}

\section{1 势能和总物种数的演化}

图 2 是初始分子数为 $64 \mathrm{ONC}$ 超胞系统在不同 温度条件下势能随时间变化的演化曲线。势能在 不同温度下, 具有相似的演化趋势。在极短的时 
间(约为 $0.3 \mathrm{ps}$ ) 内势能有个小幅上升, 然后快速下 降, 最后趋于平衡, 这是因为系统在极短的时间 内吸热, 然后 ONC 发生热分解放热反应导致势能 迅速减少且温度越高势能衰减的越快, 最终趋于 平衡。在温度 2500 和 $2700 \mathrm{~K}$ 时势能在 $0-10 \mathrm{ps}$ 时 间内衰减速度基本相同, 在 $10 \mathrm{ps}$ 之后势能在温度 为 $2700 \mathrm{~K}$ 衰减速度明显大于 $2500 \mathrm{~K}$ 条件下的衰减 速度。

图 3 是超胞系统在温度升高后总物种数随时间 变化的演化曲线。在初始时刻 $(t=0 \mathrm{ps})$, 系统只含 有 $\mathrm{ONC}$ 一种物质, 在 $3500 \mathrm{~K}$ 温度条件下, 加热到 $5 \mathrm{ps}$ 时总物种数达到最大值, 约为 90 ; 然后快速衰 减, 对比图 2 此时势能快速减少; 当加热到约 50 ps 时, 物种数减少速度减小, 势能也趋于稳定; 在较低温度条件下, 总物种数快速增加到最大值 (约为 80 ) 后, 物种数出现一个平衡阶段, 温度越高 平衡维持的时间越短; 然后物种数开始减少, 当 加热到 $100 \mathrm{ps}$ (在 $T=2700 \mathrm{~K}$ 温度条件下)时, 物种 数减少速度减小。从图 3 可以看出, $\mathrm{ONC}$ 在高温 热分解的初始阶段, 温度越高 $\mathrm{ONC}$ 热分解释放能 量的速率越快, 物种数增加越快且产生的物种数 也越多; 然后物种数出现一个短暂平衡阶段, 温
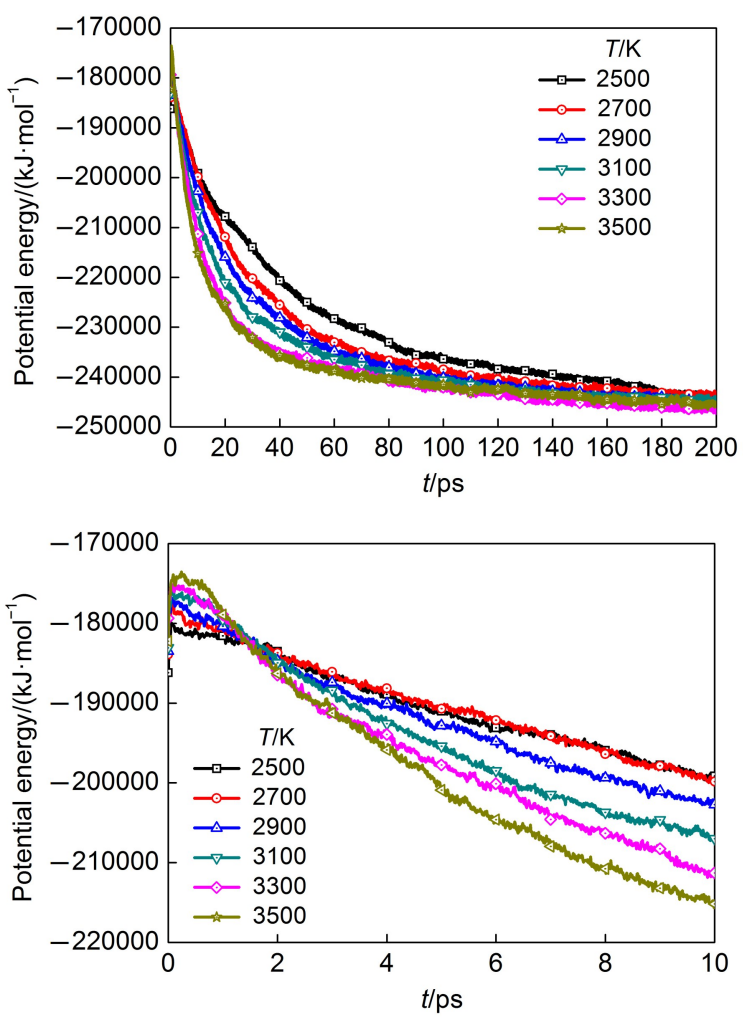

图 2 系统势能的历时曲线

Fig.2 Time evolution of potential energy for the system

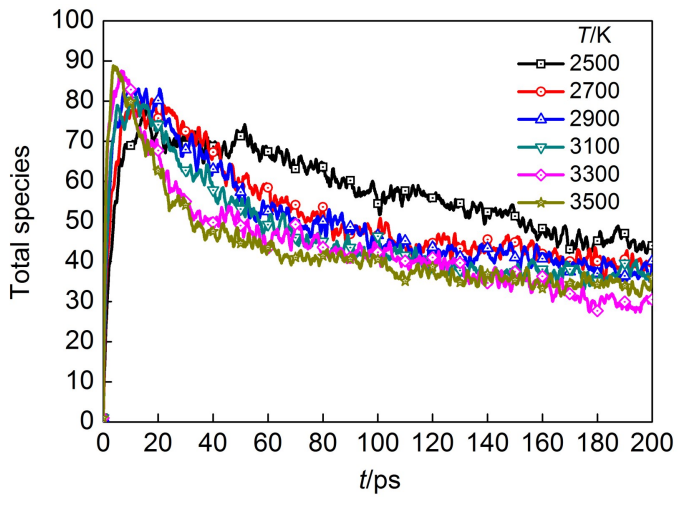

图 3 系统总物种的历时曲线

Fig.3 Time evolution of total species for the system

度越高物种数维持平衡时间越短; 然后物种数快 速衰减, 温度越高衰减越快; 在不同温度条件下 最后物种数均维持在 45 左右。

\subsection{ONC 单个晶胞的初始反应路径及热分解产物}

通过对 ONC 单个晶胞的热分解进行分析, 在 $2500 \mathrm{~K}$ 的条件下, 通过分析原子成键情况, 发现 $\mathrm{ONC}$ 初始反应路径主要有三种, 图 4 为三种路径 的演化过程示意图。在不同反应路径中, 都是笼 状结构中的 $\mathrm{C}-\mathrm{C}$ 键最先发生断裂, 这与 Owens ${ }^{14}$ 和张骥 ${ }^{2}$ 采用半经验公式计算得到的 $\mathrm{C}-\mathrm{C}$ 键比 $\mathrm{C}-$ $\mathrm{NO}_{2}$ 弱, 且 $\mathrm{C}-\mathrm{C}$ 键离解能最小的结果相一致。

从图 4(a)可以看出, ONC 分子笼状结构中的 $\mathrm{C}-\mathrm{C}$ 首先发生断裂, 然后, 笼状结构中与首先断 裂的 $\mathrm{C}-\mathrm{C}$ 键垂直且不相邻的 $\mathrm{C}-\mathrm{C}$ 键发生断裂; 随后, 笼状结构中与第一个断裂的 $\mathrm{C}-\mathrm{C}$ 键不相邻 且与第二个断裂的 $\mathrm{C}-\mathrm{C}$ 键平行的 $\mathrm{C}-\mathrm{C}$ 键发生断 裂; 再接着, 与第一个断裂的 $\mathrm{C}-\mathrm{C}$ 键平行且与另 外两个已经断裂的 $\mathrm{C}-\mathrm{C}$ 键不相邻的 $\mathrm{C}-\mathrm{C}$ 键发生 断裂, 最后形成八硝基环辛烯。

从图 4(b) 可以看出, 首先 $\mathrm{ONC}$ 分子中的 $\mathrm{C}-\mathrm{C}$ 发生断裂, 然后, 笼状结构中与已经断裂的 $\mathrm{C}-\mathrm{C}$ 键在同一平面内且平行的 $\mathrm{C}-\mathrm{C}$ 键迅速断裂, 形成 八硝基环辛四烯; 紧随其后, 笼状结构中与第一 个断裂的 $\mathrm{C}-\mathrm{C}$ 键不相邻且垂直的两个 $\mathrm{C}-\mathrm{C}$ 键先 后发生了断裂, 形成八硝基环辛烯。在此之后, 就是 $\mathrm{C}-\mathrm{NO}_{2}$ 中的 $\mathrm{C}-\mathrm{N}$ 键发生断裂。八硝基环辛 烯也是高度对称结构, 然后八硝基环辛烯中的 $\mathrm{C}-$ $\mathrm{NO}_{2}$ 中的 $\mathrm{C}-\mathrm{N}$ 键发生断裂, 形成 $\mathrm{NO}_{2}^{*}$ 自由基。

从图 4(c) 可以看出, $\mathrm{ONC}$ 分子中的 $\mathrm{C}-\mathrm{C}$ 发生 断裂后, 笼状结构中与第一个断裂的 $\mathrm{C}-\mathrm{C}$ 键垂直 且不相邻的 $\mathrm{C}-\mathrm{C}$ 键迅速断裂; 然后, 分别与两个 
(a)

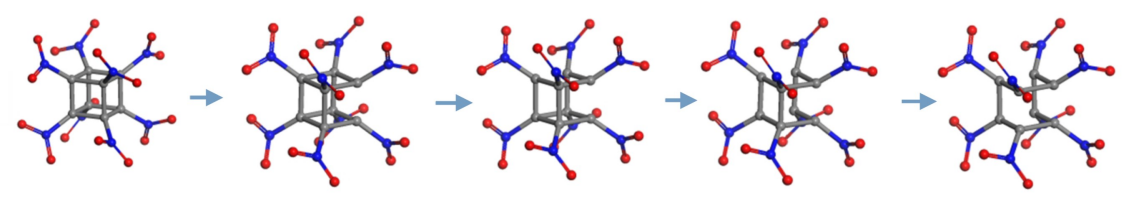

(b)

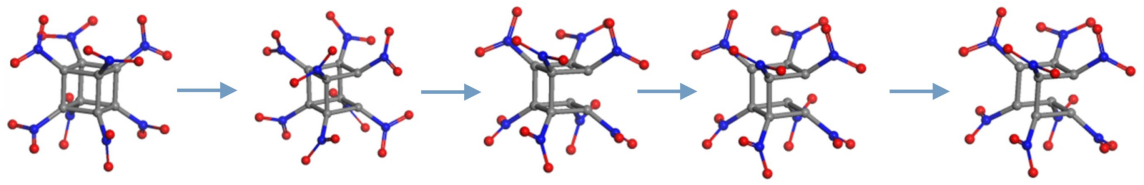

(c)

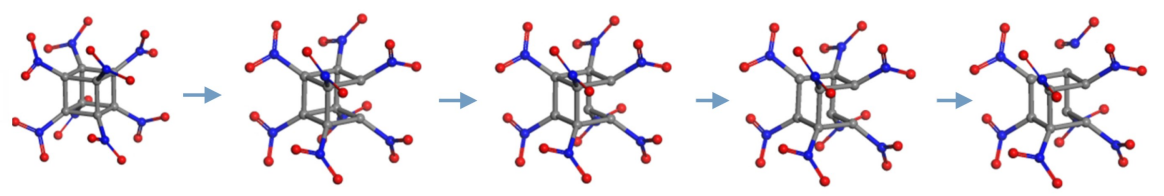

图 4 ONC 起始反应路径示意图

Fig.4 Schematic diagram of initial reaction pathways for ONC

已经断裂的 $\mathrm{C}-\mathrm{C}$ 键垂直且不相邻的 $\mathrm{C}-\mathrm{C}$ 键断 裂。在这个新分子中，与三个碳原子相连的 $\mathrm{C}$ 上的 硝基从分子中分离形成 $\mathrm{NO}_{2}^{*}$ 自由基。

这三种 $\mathrm{ONC}$ 的起始反应路径, 都是 $\mathrm{ONC}$ 分子 中的笼状结构先发生破坏, 然后才出现 $\mathrm{NO}_{2}$ 以及 $\mathrm{C}-\mathrm{NO}_{2}$ 中的 $\mathrm{O}$ 出现分离。

通过分析单个晶胞在 $2500 \mathrm{~K}$ 温度条件下前 20 ps 热分解过程得到其热分解路径, 如图 5 所示。分 析发现, $\mathrm{ONC}$ 分子中的 $\mathrm{NO}_{2}$ 以及 $\mathrm{O}$ 首先分离出 来, 生成 $\mathrm{C}_{8} \mathrm{~N}_{7} \mathrm{O}_{14} 、 \mathrm{C}_{8} \mathrm{~N}_{7} \mathrm{O}_{12} 、 \mathrm{C}_{8} \mathrm{~N}_{7} \mathrm{O}_{11} 、 \mathrm{C}_{8} \mathrm{~N}_{4} \mathrm{O}_{6}$ 以及 $\mathrm{C}_{8} \mathrm{~N}_{8} \mathrm{O}_{14}$ 等碎片, 然后他们继续发生分解, $\mathrm{CC}$ 链、
环断裂, 生成最终产物 $\mathrm{CO}_{2}$ 以及其他小分子 $\mathrm{CNO} 、 \mathrm{C}_{2} \mathrm{NO}_{2} 、 \mathrm{CNO}_{2} 、 \mathrm{NO}_{2}$ 和 $\mathrm{NO}_{3}$ 等。

\subsection{ONC 在高温条件下热分解产物分析}

图 6 是超胞系统在不同温度条件下热分解随时 间的演化曲线。从图 6 中可以看出, 在 $2500 \mathrm{~K}$ 温 度条件下, ONC 在 $5 \mathrm{ps}$ 内完全分解; 在 $3500 \mathrm{~K}$ 温 度条件下, $\mathrm{ONC}$ 在 $1.5 \mathrm{ps}$ 内完全分解, 由此可见温 度越高 $\mathrm{ONC}$ 分子分解越快。

图 7 是超胞系统热分解的主要产物随时间变化 的演化曲线。图 7 显示, $\mathrm{ONC}$ 热分解的主要产物 为 $\mathrm{O}_{2} 、 \mathrm{CO}_{2} 、 \mathrm{~N}_{2} 、 \mathrm{NO}_{2} 、 \mathrm{NO}_{3} 、 \mathrm{NO} 、 \mathrm{CO}$ 和 $\mathrm{CNO}$

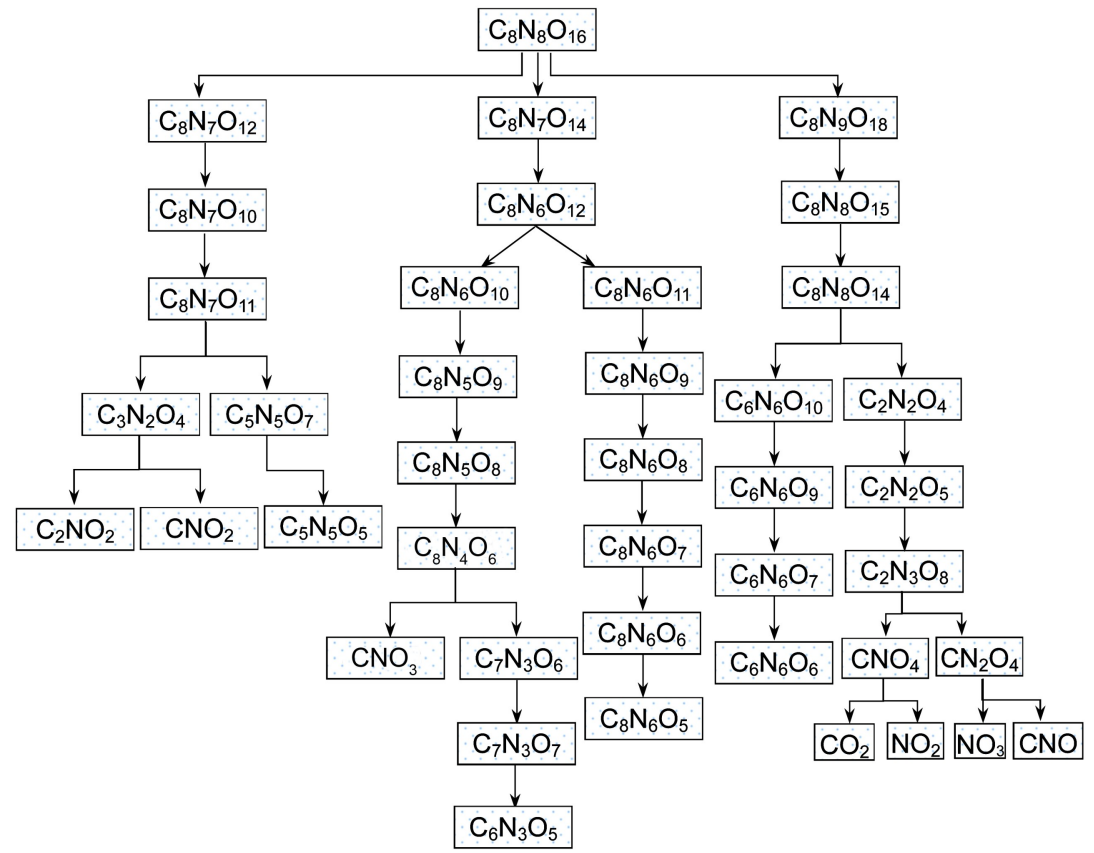

图 $5 \mathrm{ONC}$ 的热分解路径

Fig.5 Pyrolysis pathways for $\mathrm{ONC}$ 


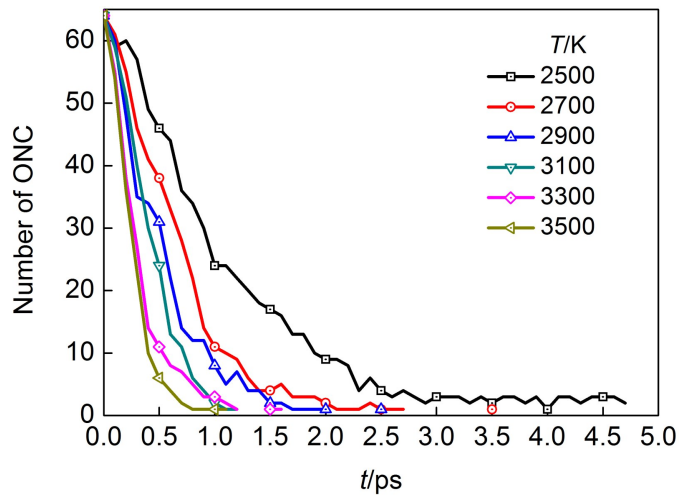

图 6 不同温度下 $\mathrm{ONC}$ 的历时曲线

Fig.6 Time evolution of $\mathrm{ONC}$ at various temperatures

等。通过分析, 在笼状结构中的 $\mathrm{C}-\mathrm{C}$ 键相继断裂 导致笼状结构发生破坏后, $\mathrm{C}-\mathrm{NO}_{2}$ 中的 $\mathrm{C}-\mathrm{N}$ 键 断裂生成 $\mathrm{NO}_{2}, \mathrm{NO}_{2}$ 是最早出现的小分子产物。从
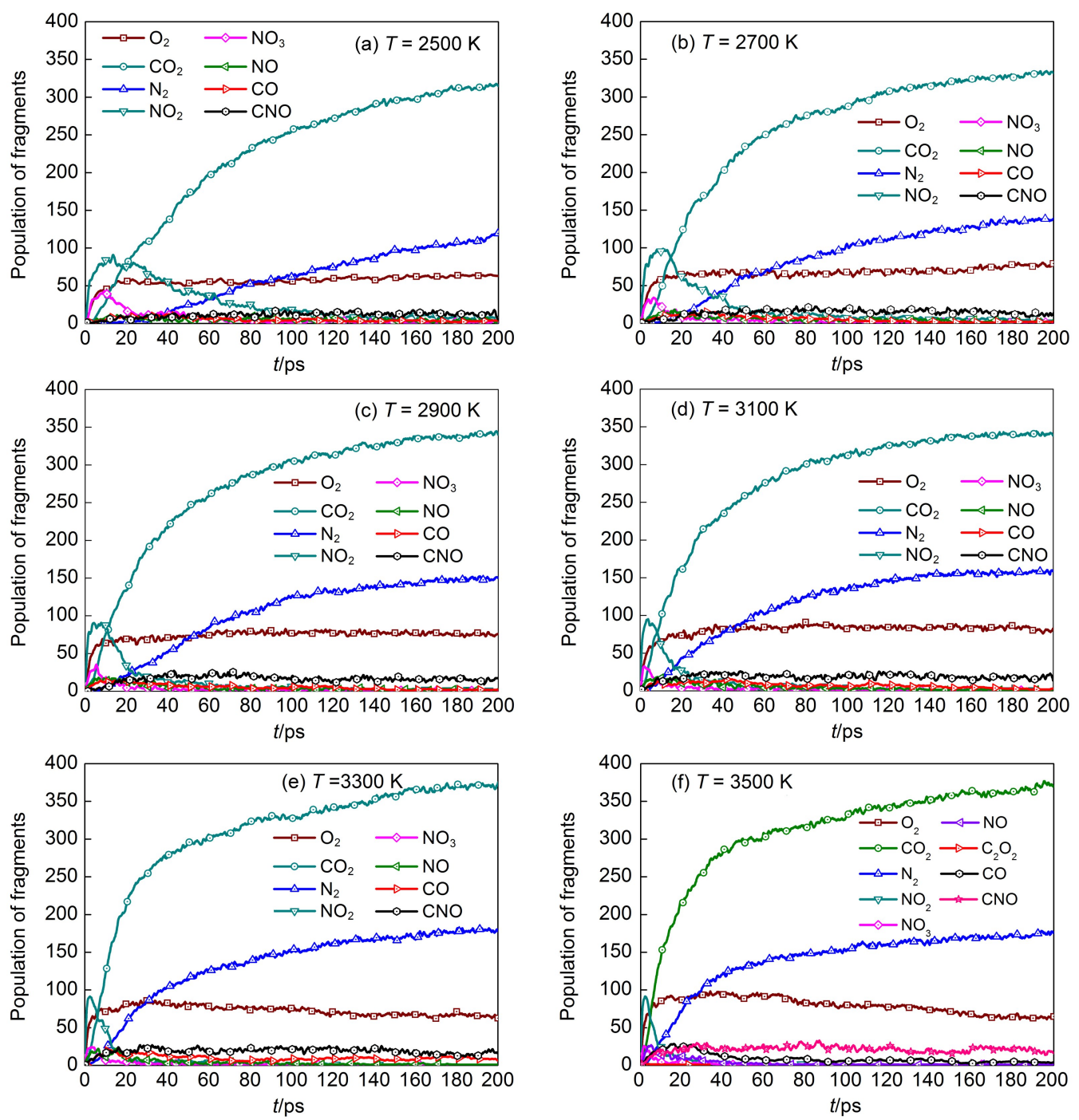

图 7 不同温度下主要产物历时曲线

Fig.7 Time evolution of main products at various temperatures 
显示的 $\mathrm{NO}_{3}$ 的产生滞后于 $\mathrm{NO}_{2}$ 相符, 而且 $\mathrm{NO}_{3}$ 数量 的最大值约为 $\mathrm{NO}_{2}$ 的最大值的 $1 / 3$ 。

$\mathrm{O}_{2}$ 也是反应过程中的主要产物之一, 在 $\mathrm{ONC}$ 中的 $\mathrm{C}-\mathrm{NO}_{2}$ 和 $\mathrm{C}-\mathrm{N}-\mathrm{O}$ 中 $\mathrm{N}-\mathrm{O}$ 键断裂分离出自
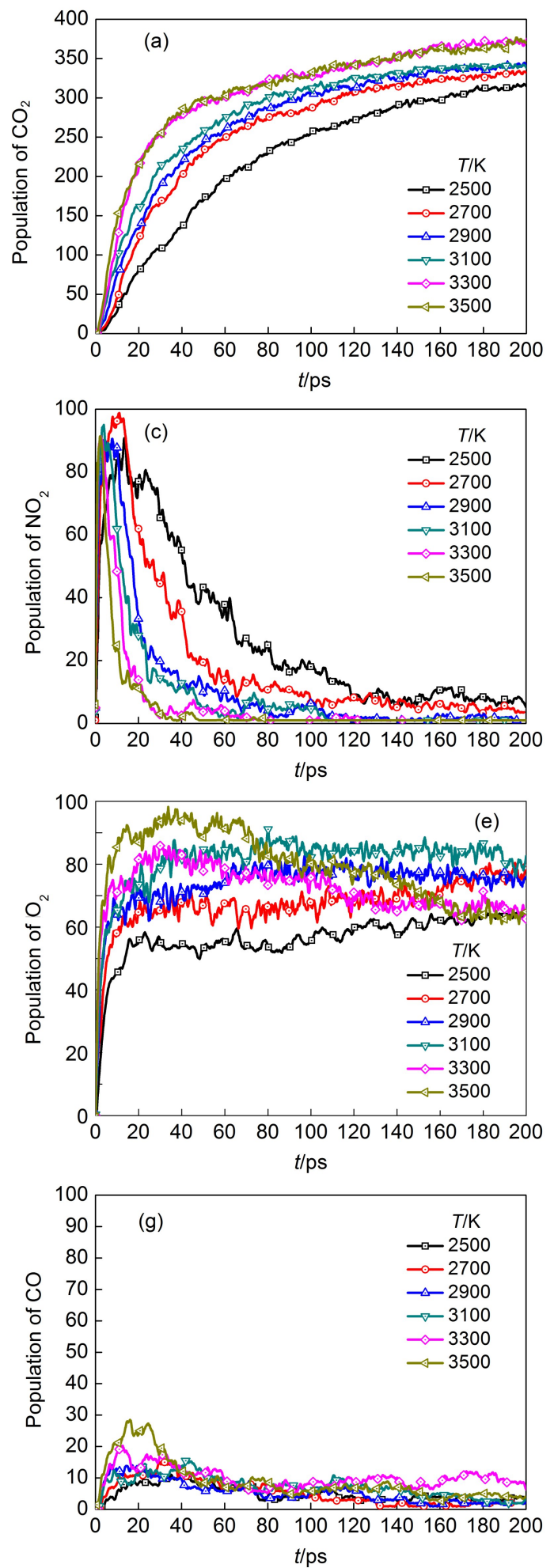

由 $\mathrm{O}$, 然后自由 $\mathrm{O}$ 形成 $\mathrm{O}_{2}$ 。在反应起始时, $\mathrm{O}_{2}$ 数量 急剧增多, 当 $\mathrm{NO}_{2}$ 的数量达到最大值时, $\mathrm{O}_{2}$ 不再增 加维持在稳定的数量直至模拟结束。 $\mathrm{CO}_{2}$ 和 $\mathrm{N}_{2}$ 是最 终产物, 当 $\mathrm{ONC}$ 分子接近被完全消耗时 $\mathrm{CO}_{2}$ 开始
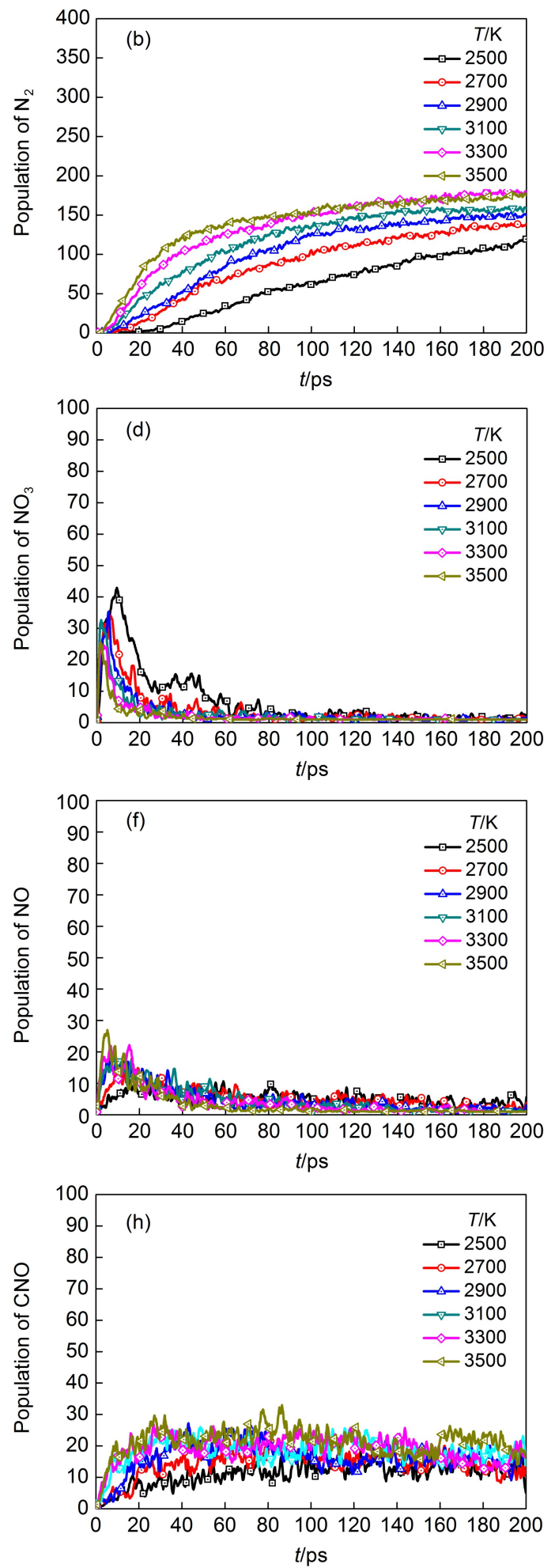

图 8 不同温度下主要产物的分布曲线

Fig.8 Time evolution of main products at various temperatures 
出现, 在模拟时间内 $\mathrm{CO}_{2}$ 数量逐渐增多, 增长速率 逐渐减小; 当 $\mathrm{NO}_{2}$ 的数量达到最大值时 $\mathrm{N}_{2}$ 数量开 始增加, 且随时间的增加逐渐增加。

\section{4 主要产物在不同温度条件下的分布}

图 8 是超胞在不同温度条件下 $\mathrm{ONC}$ 热分解过 程主要产物的对比分布曲线图。从图中可以看 出, 不同温度条件下, 在有限的模拟时间内, $\mathrm{CO}_{2}$ 和 $\mathrm{N}_{2}$ 的数量随时间均逐渐增多, 温度越高 $\mathrm{CO}_{2}$ 和 $\mathrm{N}_{2}$ 出现的越早且产生的速率越大, 且在温度较低 的条件下, 温度对 $\mathrm{CO}_{2}$ 和 $\mathrm{N}_{2}$ 的产生影响更大, 当 温度为 3300 和 $3500 \mathrm{~K}$ 时, 在 $20 \mathrm{ps}$ 后两种温度下 $\mathrm{CO}_{2}$ 的数量趋于一致, 而在 $100 \mathrm{ps}$ 左右时两种温度 下 $\mathrm{N}_{2}$ 的数量趋于一致。随着温度的升高, 产生 $\mathrm{N}_{2}$ 的时间越早，但是 $\mathrm{N}_{2}$ 的产生时间明显滞后于 $\mathrm{CO}_{2}$; 对比图 8(c) 可以发现, 在 $\mathrm{NO}_{2}$ 达到最大值时, $\mathrm{N}_{2}$ 开 始出现并快速增多。 $\mathrm{NO}_{2}$ 和 $\mathrm{NO}_{3}$ 是最为活跃的中间 产物, 在不同温度条件下具有相似的演化趋势, 在 $\mathrm{ONC}$ 分解过程的初期 $\mathrm{NO}_{2}$ 和 $\mathrm{NO}_{3}$ 的数量急剧增 加, 当达到最大值后开始快速衰减, 温度越高到 达峰值的时间越短。通过对比发现, $\mathrm{NO}_{3}$ 的产生滞 后于 $\mathrm{NO}_{2}$ 且 $\mathrm{NO}_{3}$ 的峰值大幅低于 $\mathrm{NO}_{2}$ 的峰值, 在增 长阶段 $\mathrm{NO}_{3}$ 的增长速率低于 $\mathrm{NO}_{2}$ 的增长速率, 而在 衰减阶段 $\mathrm{NO}_{3}$ 的衰减速率高于 $\mathrm{NO}_{2}$ 的衰减速率。衰 减是由于 $\mathrm{NO}_{2}$ 和 $\mathrm{NO}_{3}$ 参与反应生成 $\mathrm{N}_{2}$ 而不断的被消 耗, 衰减速率随温度的升高增大, 在较高温度条 件下, 有限的模拟时间内 $\mathrm{NO}_{2}$ 和 $\mathrm{NO}_{3}$ 已经被完全消 耗。从图 8(e)中可以看出, 在反应起始阶段, $\mathrm{O}_{2}$ 的 数量急剧增大且温度越高 $\mathrm{O}_{2}$ 产生速率越大, 在较 低温度的条件下, $\mathrm{O}_{2}$ 的数量达到最大值后维持稳 定, 而在较高温度 $(3300$ 和 $3500 \mathrm{~K})$ 条件下, $\mathrm{O}_{2}$ 的数 量达到最大值后开始衰减。

$\mathrm{NO}$ 也是比较活跃的中间产物之一, 从图 8(f) 可以看出, 在有限模拟时间内 $\mathrm{NO}$ 的数量演化趋势 先增大后较少, 在增长阶段, $\mathrm{NO}$ 的产生速率随温 度的升高而增大, $\mathrm{NO}$ 的产生速率比 $\mathrm{NO}_{2}$ 和 $\mathrm{NO}_{3}$ 的 产生速率小, 且温度越低时越显著; 在衰减阶 段, $\mathrm{NO}$ 的衰减速率受温度影响较小。通过分析化 学键的成断键情况得知 $\mathrm{NO}$ 主要由三种途径产生, (1) $\mathrm{NO}_{2}+\mathrm{C}-\mathrm{R} \rightarrow \mathrm{R}-\mathrm{C}-\mathrm{O}-\mathrm{NO}$, 然后 $\mathrm{O}-\mathrm{N}$ 键 断裂生成 $\mathrm{NO}$, 这与 $\mathrm{O}-\mathrm{NO}$ 键的离解能较小一 致; (2) $\mathrm{R}-\mathrm{C}-\mathrm{N}-\mathrm{O}$ 中的 $\mathrm{C}-\mathrm{N}$ 键断裂生成 $\mathrm{NO}$; (3) 由 $\mathrm{NO}_{2}$ 和 $\mathrm{NO}_{3}$ 等相互反应生成 $\mathrm{NO}$ 。

\section{4 结 论}

采用 ReaxFF 反应力场对 $\mathrm{ONC}$ 的热分解过程进 行了模拟。研究表明 $\mathrm{ONC}$ 的触发反应是分子中笼 状结构中的 $\mathrm{C}-\mathrm{C}$ 键断裂。 $\mathrm{ONC}$ 在热分解过程中笼 状结构首先发生破坏, 发现 $\mathrm{ONC}$ 分子中的笼状结 构发生破坏的三种不同路径, 在此之后 $\mathrm{NO}_{2}$ 和 $\mathrm{C}-$ $\mathrm{NO}_{2}$ 中的 $\mathrm{O}$ 分离出来。这与许多高能化合物 (如 TNT、RDX、HMX 和 CL-20)在热分解过程中均从 $\mathrm{C}-\mathrm{NO}_{2}$ 中的 $\mathrm{C}-\mathrm{N}$ 键首先发生断裂完全不同, 这 可能是因为 $\mathrm{ONC}$ 就有较高的应变能。

通过对热分解过程产物分析可知, $\mathrm{ONC}$ 在高 温条件下热分解的主要产物有 $\mathrm{NO}_{2} 、 \mathrm{NO}_{3} 、 \mathrm{NO}$ 、 $\mathrm{O}_{2} 、 \mathrm{CO}_{2} 、 \mathrm{~N}_{2} 、 \mathrm{CNO}$ 以及 $\mathrm{CO}$ 等。 $\mathrm{NO}_{2} 、 \mathrm{NO}_{3}$ 和 $\mathrm{NO}$ 是重要的早期产物, $\mathrm{NO}_{2}$ 主要来自于 $\mathrm{C}-\mathrm{NO}_{2}$ 中 $\mathrm{C}-\mathrm{N}$ 键断裂产生的; $\mathrm{NO}_{3}$ 主要来自于 $\mathrm{NO}_{2}$ 和 $\mathrm{O}$ 结 合以及 $\mathrm{C}-\mathrm{NO}_{3}$ 中 $\mathrm{C}-\mathrm{N}$ 键断裂产生的; $\mathrm{NO}$ 主要来 自于 $\mathrm{NO}_{2}+\mathrm{C}-\mathrm{R} \rightarrow \mathrm{R}-\mathrm{C}-\mathrm{O}-\mathrm{NO}$, 然后 $\mathrm{O}-\mathrm{N}$ 键 断裂生成 $\mathrm{NO}, \mathrm{R}-\mathrm{C}-\mathrm{N}-\mathrm{O}$ 中的 $\mathrm{C}-\mathrm{N}$ 键断裂生 成 $\mathrm{NO}$ 以及次级反应生成 $\mathrm{NO}$ 。产生速率大小顺序 为: $\mathrm{NO}_{2}>\mathrm{NO}_{3}>\mathrm{NO}, \mathrm{NO}_{3}$ 和 $\mathrm{NO}$ 在产量上明显低 于 $\mathrm{NO}_{2}$, 在不同温度条件下 $\mathrm{NO}_{2} 、 \mathrm{NO}_{3}$ 和 $\mathrm{NO}$ 演化 趋势相似。在有限的模拟时间内 $\mathrm{O}_{2} 、 \mathrm{CO}_{2} 、 \mathrm{~N}_{2}$ 是终 态产物, $\mathrm{CO}_{2} 、 \mathrm{~N}_{2}$ 的数量随时间增加逐渐增大, $\mathrm{N}_{2}$ 的产生明显滞后于 $\mathrm{CO}_{2}$, 温度对 $\mathrm{O}_{2}$ 的演化过程影响 显著。温度对各种产物的产生均有不同程度的影 响。该研究为 $\mathrm{ONC}$ 的热分解过程提供了详细的反 应细节, 揭示了 $\mathrm{ONC}$ 热分解机理, 为 $\mathrm{ONC}$ 的实际 应用提供参考。

\section{References}

(1) Qiu, L.; Xu, X. J.; Xiao, H. M. Chin. J. Energy Mater. 2005, 13, 262. [邱 玲, 许晓娟, 肖鹤鸣. 含能材料, 2005, 13, 262.]

(2) Zhang, J. Quantum Chemical Studies on the Structures and Properties of Organic Caged Energetic Compounds Including Polynitrocubanes. Ph. D. Dissertation, Nanjing University of Science and Technology, Nanjing, 2003. [张 瀷. 多硝基立方 烷等有机笼状高能化合物结构和性能的量子化学研究 [D]. 南京: 南京理工大学, 2003.]

(3) Ji, Y. P.; Wang, B. Z.; Zhang, Z. Z.; Lu, Q.; Zhu, C. H. Chin. J. Energy Mater. 2004, 12, 189. [姬月萍, 王伯周, 张志忠, 刘 惩, 朱春华. 含能材料, 2004, 12, 189.]

(4) Eaton, P. E.; Cole, T. W., Jr. J. Am. Chem. Soc. 1964, 86, 3157. doi: 10.1021/ja01069a041

(5) Eaton, P. E.; Cole, T. W., Jr. J. Am. Chem. Soc. 1964, 86, 962. doi: 10.1021/ja01059a072 
(6) Lukin, K.; Li, J. C.; Gilardi, R.; Eaton, P. E. Angew. Chem. Int. Edit. 1996, 35, 864. doi: 10.1002/anie.199608641

(7) Lukin, K.; Li, J. C.; Gilardi, R.; Eaton, P. E. Angew. Chem. Int Edit. 1996, 35, 866. doi: 10.1002/anie.199608661

(8) Lukin, K. A.; Li, J. C.; Eaton, P. E.; Gilardi, R. J. Org. Chem. 1997, 62, 8490. doi: 10.1021/jo971308k

(9) Zhang, M. X.; Eaton, P. E.; Gilardi, R. Angew. Chem. Int. Edit 2000, 39, 401. doi: 10.1002/(SICI)1521-3757(20000117)112: $2<422::$ AID-ANGE422>3.0.CO;2-2

(10) Richard, R. M.; Ball, D. W. J. Hazard. Mater. 2009, 164, 1595. doi: 10.1016/j.jhazmat.2008.09.078

(11) Richard, R. M.; Ball, D. W. J. Hazard. Mater. 2009, 164, 1552. doi: 10.1016/j.jhazmat.2008.08.057

(12) Peköz, R.; Erkoç, Ş. Comput. Mater. Sci. 2009, 46, 849. doi: 10.1016/j.commatsci.2009.04.020

(13) Chi, W. J.; Li, L. L.; Li, B. T.; Wu, H. S. J. Mol. Model. 2013, 19, 571. doi: 10.1007/s00894-012-1582-1

(14) Owens, F. J. J. Mol. Struct. 1999, 460, 137. doi: 10.1016/ S0166-1280(98)00312-1

(15) Chi, W.; Wang, X. Y.; Li, B. T.; Wu, H. S. J. Mol. Model. 2012, 18, 4217. doi: 10.1007/s00894-012-1430-3

(16) Li, J. S. Theor. Chem. Acc. 2009, 122, 101. doi: 10.1007/ s00214-008-0489-5

(17) Liu, L. C.; Bai, C.; Sun, H.; Goddard, W. A., III. J. Phys. Chem. A 2011, 115, 4941. doi: 10.1021/jp110435p
(18) Zhan, J. H.; Wu, R. C.; Liu, X. X.; Gao, S. Q.; Xu, G. G. Fuel 2014, 134, 283. doi: 10.1016/j.fuel.2014.06.005

(19) Ghenoweth, K.; van Duin, A. C. T.; Dasgupta, S.; Goddard, W. A., III. J. Phys. Chem. A 2009, 113, 1740. doi: 10.1021/ jp8081479

(20) Cheung, S.; Deng, W. Q.; van Duin, A. C. T.; Goddard, W. A., III. J. Phys. Chem. A 2005, 109, 851. doi: 10.1021/jp0460184

(21) Mueller, J. E.; van Duin, A. C. T.; Goddard, W. A., III. J. Phys. Chem. C 2010, 114, 4939. doi: 10.1021/la4006983

(22) Kim, S. Y.; Kumar, N.; Persson, P.; Sofo, J.; van Duin, A. C. T.; Kubicki, J. D. Langmuir 2013, 29, 7838. doi: 10.1021/ la4006983

(23) Strachan, A.; Kober, E. M.; van Duin, A. C. T.; Oxgaard, J.; Goddard, W. A. J. Chem. Phys. 2005, 122, 54502. doi: $10.1063 / 1.1831277$

(24) Liu, H.; Dong, X.; He, Y. H. Acta Phys. -Chim. Sin. 2014, 30, 232. [刘 海, 董 晓, 何远航. 物理化学学报, 2014, 30, 232.] doi: 10.3866/PKU.WHXB201312101

(25) Liu, H.; Li, Q. K.; He, Y. H. Acta Phys. Sin. 2013, 62, 1. [刘 海, 李启楷, 何远航. 物理学报, 2013, 62, 1.] doi: 10.7498/aps.62.208202

(26) Zhou, T. T.; Huang, F. L. J. Phys. Chem. B 2011, 115, 278. doi: $10.1021 /$ jp $105805 \mathrm{w}$

(27) http://lammps.sandia.gov/ (accessed Nov 16, 2015). 